\section{Response}

I am truly grateful and honored to receive this prize, and I am equally delighted to receive it for this particular topic, which is very close to my heart.

All my professional career so far has been spent on the interface between computer science and mathematics, and I genuinely believe that certain amusing cultural differences between the two communities look really insignificant when compared to the amount of inspiration and fresh and novel ideas their interaction brings to both disciplines. In a sense, the work I am being awarded for is a quintessence of this philosophy. In order to be able to do something really computational (computer-aided theorem proving in extremal combinatorics using packages for semidefinite programming), one has to be able to reveal and understand fundamental, albeit somewhat simple by mathematical standards, algebraic and analytical structures behind this activity and be guided by them. Thank you again, both for the recognition of my own contribution and for promoting this important interdisciplinary ideology!

I would like to use this opportunity and thank all institutions I have been fortunate to be affiliated with (Steklov Mathematical Institute, IAS, University of Chicago, Toyota Technological Institute) for the stimulating intellectual environment that encouraged work on difficult and interesting problems. I am very grateful to my own collaborators on the project (Hamed Hatami, Jan Hladky, Daniel Kral, Sergei Norin, Oleg Pikhurko), as well as to many other young "flag algebraists" for developing this theory. Last but not the least my special thanks go to my wife, Iren, and my children, Andrew and Maria, for humorously bearing with the half-absence of their husband and daddy even when he appears to be fully present physically.

\section{About the Prize}

The Robbins Prize was established in 2005 in memory of David P. Robbins by members of his family. Robbins, who died in 2003, received his Ph.D. in 1970 from the Massachusetts Institute of Technology. He was a long-time member of the Institute for Defense Analysis Center for Communications Research and a prolific mathematician whose work (much of it classified) was in discrete mathematics.

The prize is given for a paper that (1) reports on novel research in algebra, combinatorics, or discrete mathematics; (2) has a significant experimental component; (3) is on a topic broadly accessible; and (4) provides a simple statement of the problem and clear exposition of the work. The US $\$ 5,000$ prize is awarded every three years.

The Robbins Prize is awarded by the AMS Council acting on the recommendation of a selection committee. For the 2013 prize, the members of the selection committee were: Daniel J. Allcock, J. P. Buhler, Maria Chudnovsky, Bernd Sturmfels, and Benjamin Sudakov.

The previous recipients of the Robbins Prize are Samuel Ferguson and Thomas C. Hales (2007) and Ileana Streinu (2010).

-Elaine Kehoe

\title{
2013 Satter Prize
}

MARYAM MIRZAKHANI received the 2013 AMS Ruth Lyttle Satter Prize in Mathematics at the 119th Annual Meeting of the AMS in San Diego, California, in January 2013.

\section{Citation}

The 2013 Ruth Lyttle Satter Prize in Mathematics is awarded to Maryam Mirzakhani for her deep contributions to the theory of moduli spaces of Riemann surfaces.

Her earliest work, the topic of her thesis, was a volume formula for the moduli space of bordered Riemann surfaces of genus $g$ with $n$ geodesic boundary components, a formula that expresses this volume as a polynomial in the lengths of the

DOI: http://dx.doi.org/10.1090/noti969 boundary components. That there exists a formula of this nature was itself surprising, but more surprising were the results she was able to extract from it: a new proof of the celebrated conjecture of Witten on the intersection numbers of tautology classes on moduli space and, in a completely different direction, an asymptotic formula for the lengths of simple closed geodesics on a compact hyperbolic surface.

Much of her work subsequent to this has focused on the Teichmüller dynamics of moduli space. In particular, she was able to construct a measure-preserving conjugacy between Thurston's earthquake flow on Teichmüller space and horocycle flow on the associated space of quadratic differentials and as an immediate and long sought-after consequence of this to prove that earthquake flow 


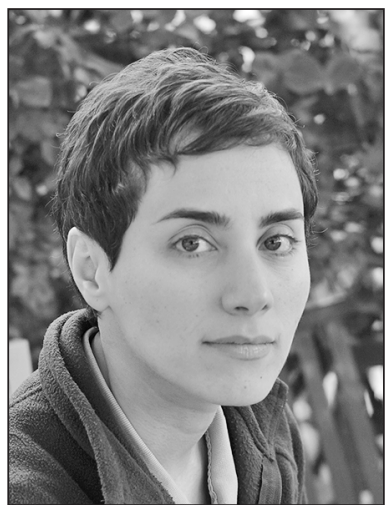

Maryam Mirzakhani is ergodic. In another vein, her recent work with Eskin establishes striking analogues for the Teichmüller flow and the mapping class group of Selberg's classical "Prime Geodesic Theorem" for the modular surface and the modular group. Moreover, in a work in progress, they have unearthed some unexpected and intriguing analogues, in this Teichmüller setting, of the Ratner unipotent rigidity theorems in homogeneous dynamics.

\section{Biographical Sketch}

Maryam Mirzakhani grew up in Tehran, Iran. She obtained her B.Sc. in mathematics (1999) from the Sharif University of Technology. She holds a Ph.D. from Harvard University (2004), where her advisor was Curtis McMullen. From 2004 to 2008 she was a Clay Mathematics Institute Research Fellow and an assistant professor at Princeton University. She is currently a professor at Stanford University. Her research interests include Teichmüller theory, hyperbolic geometry, and ergodic theory.

\section{Response}

I am deeply honored to receive the Ruth Lyttle Satter Prize. This would not have been possible without many people who helped me along. I am grateful to my collaborators and colleagues who helped me all these years. I would like to thank my great teachers in Iran, both in high school and at Sharif University, for providing a stimulating environment for their students. All these opportunities and the people who made them possible, regardless of the difficulties of the times, deserve my sincere gratitude. I am also grateful to my Ph.D. advisor, Curt McMullen, for his unceasing support and for introducing me to fascinating areas of mathematics.

I have enjoyed a pleasant and supportive environment during my time at Harvard, Princeton, and Stanford. Still, in my opinion, the situation of women in math is far from ideal. The social barriers for girls who are interested in mathematical sciences might not be lower now than they were when I grew up. And balancing career and family remains a big challenge. It makes most women face difficult decisions which usually compromise their work. However, there has been a lot of progress over the years, and I am sure this trend will continue.

Finally, I would like to thank my friends who have been like my family away from home. I am grateful to my husband, Jan, for being my best friend and companion and for encouraging me when I need it the most. I would like to thank my parents, who always believed in me and let me be who I am. They have been my inspiration throughout my life.

\section{About the Prize}

The Satter Prize is awarded every two years to recognize an outstanding contribution to mathematics research by a woman in the previous six years. Established in 1990 with funds donated by Joan S. Birman, the prize honors the memory of Birman's sister, Ruth Lyttle Satter. Satter earned a bachelor's degree in mathematics and then joined the research staff at AT\&T Bell Laboratories during World War II. After raising a family, she received a Ph.D. in botany at the age of forty-three from the University of Connecticut at Storrs, where she later became a faculty member. Her research on the biological clocks in plants earned her recognition in the United States and abroad. Birman requested that the prize be established to honor her sister's commitment to research and to encourage women in science. The prize carries a cash award of US $\$ 5,000$.

The Satter Prize is awarded by the AMS Council acting on the recommendation of a selection committee. For the 2013 prize, the members of the selection committee were Victor W. Guillemin, Svetlana Y. Jitomirskaya, and Raman Parimala.

Previous recipients of the Satter Prize are: Dusa McDuff (1991), Lai-Sang Young (1993), Sun-Yung Alice Chang (1995), Ingrid Daubechies (1997), Bernadette Perrin-Riou (1999), Karen E. Smith (2001), Sijue Wu (2001), Abigail Thompson (2003), Svetlana Jitomirskaya (2005), Claire Voisin (2007), Laure Saint-Raymond (2009), and Amie Wilkinson (2011).

_Elaine Kehoe 